\title{
Functionality and Collaboration Mechanisms for Cognitive Wireless Networks and Systems
}

\author{
Panagiotis Demestichas • Klaus Moessner • \\ Mikhail Smirnov $\cdot$ Didier Bourse
}

Published online: 5 December 2009

(C) Springer Science+Business Media, LLC 2009

The unparalleled evolution of wireless communications is reflected in the tremendous investments on research and development, targeted at the continuous introduction of innovations that could serve the information society. This has led to the coexistence and complementary exploitation of versatile, legacy and also emerging radio access technologies (RATs), such as: wireless wide area networks (such as 2G/2.5G/3G/3G+ mobile communications, the IEEE 802.16 suite and WiMAX), broadcasting technologies, wireless short range networks (such as WLANs/WPANs and wireless sensor networks). Moreover, the wireless world is currently migrating towards the B3G (Beyond the 3rd Generation) era. In the B3G era, network operators (NOs) will have to address increased complexity due to the heterogeneity of the network and terminal infrastructure, the ever-increasing user requirements, the co-existence and need for complementary exploitation of various RATs. A NO should rely on different RATs for raising the customer satisfaction, and achieving the required quality of service (QoS) levels, cost-effectively.

We received numerous high quality contributions in this field. After impartial review, we accepted five papers. The papers selected for this special issue address research problems from terminals to networks and from Spectrum and Radio Resource Management to applications.

P. Demestichas $(\bowtie)$

Department of Digital Systems, University of Piraeus, Piraeus, Greece

e-mail: pdemest@unipi.gr

K. Moessner

Centre for Communication Systems Research, University of Surrey, Surrey, UK

M. Smirnov

Research Institute for Open Communication Systems, Fraunhofer FOKUS, Berlin, Germany

D. Bourse

Alcatel Lucent, Paris, France 
The first paper "Mobility Management Strategies in Heterogeneous Cognitive Radio Networks", by Z. Damljanovic presents the requirements for mobility management tasks, along with the mobility management protocol architecture, in order to fulfill these requirements, taking into account cross layer design, regardless the underlying radio technology.

The second paper, titled "Comprehensive Capacity Ensured Distributed Binary Power Allocation in Dense Cognitive Networks" is authored by C. Zhao and K. Kwak. The contribution of this paper is twofold; first, the paper analyses the case that the secondary nodes can detect both transmitter and receiver of the primary system. Thereafter, the case that the secondary nodes only have the information about the primary transmitter is discussed thoroughly. A general power allocation scheme, suitable for both cases is also presented and discussed.

The third paper is authored by Z. Feng, Q. Zhang, D. Fan, L. Liang and P. Zhang and is titled "Self-Configuration for Wireless Local Area Networks". This paper focuses on two key issues of self-configuration, namely network condition awareness and corresponding configuration decision, so as to minimize the complexity of the configuration of the new deployed Access Point.

The fourth paper, titled "High-Level Design Approach for the Specification of Cognitive Radio Equipments Management APIs", authored by C. Moy, asserts that it is necessary to add, apart from real-time radio adaptation and sensing capabilities, inside the cognitive radio equipments some management facilities. In the paper a high-level design approach for the specification of a management framework is proposed.

The last paper, authored by L. Lima and A. Calsavara and titled "Autonomic Application-Level Message Delivery Using Virtual Magnetic Fields", presents the use of the concept of magnetic fields borrowed from physics to enable selforganizing message forwarding on overlay networks over physical networks with possibly mobile nodes.

At the end of the editorial of this special issue, the guest editors would like to thank the authors for their contributions, and the reviewers for their time and comments. We would also like to thank the Editor-in-Chief, Prof. M. Malek, as well as Mrs M. Fearon for their support. We hope that the readers will enjoy this issue!

\section{Guest Editors}

Panagiotis Demestichas is an Associate Professor with the University of Piraeus, department of Digital Systems. Since November 2008 he has been the Technical Manager of FP7 End-to-End Efficiency $\left(E^{3}\right)$ Project. Also, since January 2004 he is the chairman of Working Group 6 (WG6) of WWRF, currently called "Cognitive Wireless Networks and Systems". His research interests include the design and performance evaluation of wireless and fixed broadband networks, software engineering, service and network management, algorithms and complexity theory, and queuing theory. He has several publications in international journals and conferences. He is a member of IEEE, ACM and of the Technical Chamber of Greece. 
Klaus Moessner is a professorial research fellow in Mobile Communications in the Centre for Communication Systems Research at the University of Surrey. His research interests include reconfigurability of the different system levels, including reconfiguration management and scheduling in wireless networks and adaptability of multimodal user interfaces. He investigates and teaches mobile service platforms, service oriented architectures, mobile service delivery and service enablers. He also works in the area of resource efficiency and on mechanisms for dynamic resource allocation. He leads a research team investigating reconfigurable radio, dynamic spectrum access and the regulatory implications of DSA and Cognitive Radio Networks.

Mikhail Smirnov is with Fraunhofer FOKUS-Research Institute for Open Communication Systems, in Berlin, Germany since 1994, as well as an Assistant Professor. His research interests are in advanced Internet services, self-organisation and self-management of network features, policy-based networking and policybased programmability. He serves the community as COMNET area editor, international conferences chair and TPC member for more than 50 conferences and journals. He is EU Commission expert in high performance networking and future and emerging technologies.

Didier Bourse currently is the Director of the European Research Cooperation in Alcatel-Lucent, France, since August 2008. Prior to this position, Dr. Bourse has worked for over 10 years in the fields of military SDR and commercial SDR/CR, at Thales Communications and at Motorola Labs in Paris. While being with Motorola he was the Technical Manager of the EC FP5 research project TRUST, and coordinated the SDR activities within the wireless world research forum (WWRF). $\mathrm{He}$ was the Project Manager of the Integrated Projects E2R (End-to-End Reconfigurability) I and II, and for the first year (2008) of $\mathrm{E}^{3}$ (End-to-End Efficiency), which is on introducing cognitive systems in the wireless world. 